Northwestern University School of Law Northwestern University School of Law Scholarly Commons

Faculty Working Papers

2008

\title{
Moral Spillovers: The Effect of Moral Violations on Deviant Behavior
}

Elizabeth Mullen

Stanford University Graduate School of Business

Janice Nadler

Northwestern University School of Law, jnadler@northwestern.edu

\section{Repository Citation}

Mullen, Elizabeth and Nadler, Janice, "Moral Spillovers: The Effect of Moral Violations on Deviant Behavior" (2008). Faculty Working Papers. Paper 149.

http://scholarlycommons.law.northwestern.edu/facultyworkingpapers/149 
Running Head: Moral Spillovers

Moral Spillovers: The Effect of Moral Violations on Deviant Behavior

\author{
Elizabeth Mullen \\ Stanford University
}

\&

Janice Nadler

Northwestern University \& American Bar Foundation

Word Count: 6,600 


\begin{abstract}
Two experiments investigated whether outcomes that violate people’s moral standards increase their deviant behavior (the moral spillover effect). In Study 1, participants read about a legal trial in which the outcome supported, opposed or was unrelated to their moral convictions. Relative to when outcomes supported moral convictions, when outcomes opposed moral convictions people judged the outcome to be less fair, were more angry, were less willing to accept the outcome, and were more likely to take a borrowed pen. In Study 2, participants who recalled another person’s moral violation were more likely to cheat on an experimental task relative to angry or neutral condition participants. Taken together, results provide evidence for moral spillover: outcomes that violate moral standards increase deviant behavior.
\end{abstract}




\section{Moral Spillovers: The Effect of Moral Violations on Deviant Behavior}

If a person sees unfairness, or illegitimacy, or unworthiness of trust in one instance, how far does his disillusionment extend? How much of his attitude spills over into other areas and into his actual behavior? (Friedman, 1975, p. 118)

Are people more willing to engage in deviant behavior in response to perceived injustice? The idea that deviant behavior can emerge from an unjust event has intuitive appeal. For example, the 1992 Los Angeles riots followed on the heels of widespread outrage regarding the acquittal of four officers accused of beating Rodney King. The riots that erupted after the verdict left 50 people dead, 2000 injured and 800 buildings burned (Cannon, 1999). Although the causes of the rioting are undoubtedly complex and involved many different factors, we propose it is plausible that one factor at play might have been that the acquittal violated many people's moral standard for what constituted a "just” outcome, leading to anger, perceptions of institutional illegitimacy and unfairness, and a greater willingness to engage in deviant behavior. The present research explores the relationship between "immoral” outcomes and deviant behavior by investigating whether outcomes or actions that violate people's moral standards increase their likelihood of engaging in deviant behavior.

\section{Moral Mandates}

When people have a strong attitude that they see as rooted in moral conviction, they have a “moral mandate” (Skitka, 2002). For example, a person who has a strong attitude about abortion (as defined by extremity, importance, and certainty; Petty \& Krosnick, 1995) would also have a moral mandate (MM) if they saw their position on abortion as tied to their core moral values. A commitment to a MM allows perceivers to classify the actions of institutions, authorities, and individuals into the mutually exclusive categories of legitimate thought or deed versus fundamental transgression (Skitka, 2002). Indeed, several studies have documented "the MM 
effect" -- that when people have a MM their sense of fairness is determined more by whether their mandated outcome is achieved than by procedural fairness (Skitka, 2002; Skitka \& Mullen, 2002). When outcomes threaten people's MMs, they respond with anger and devalue the fairness of the outcome and procedures (Mullen \& Skitka, 2006). In contrast, when outcomes support people’s MMs they perceive the outcome and procedures to be more fair (Mullen \& Skitka, 2006). Support for the MM effect has been documented across a variety of topical domains and samples, including a national random sample of Americans' reactions to the Elian Gonzalez case (Skitka \& Mullen, 2002); people's reactions to hypothetical legal and Supreme Court decisions (Mullen \& Skitka, 2006; Skitka 2002); a national random sample of Americans' reactions to the Supreme Court's decision to allow Oregon self-determination about physician assisted suicide (Skitka, 2006); and students reactions to decisions made by officials at their university (Bauman, 2007). Thus, it is well documented that people’s MMs influence their perceptions of fairness.

In the current research, we extend this prior research by exploring whether MM violations might have consequences that extend beyond perceptions of the original event and influence people’s subsequent behavior. There are several characteristics of attitudes held with moral conviction that suggest that MM violations might have behavioral consequences. First, attitudes held with moral conviction are experienced as universal truths that others should also share (i.e., they are perceived as terminal absolutes rather than personal preferences; Hare, 1981; Kant, 1786; Haidt, Rosenberg \& Hom, 2003). For example, if one has a moral conviction that gender equality is right and good, then one should perceive that gender equality is right not only in one's own culture but in other cultures as well (Haidt, Rosenberg, \& Hom, 2003). Second, moral convictions can be authority independent (see Kohlberg, 1984; Piaget, 1997; Skitka, Bauman \& Mullen, 2008). That is, moral convictions sometimes come more from within than from outside 
the person, such as from society or authorities. If, for example, one has a strong moral conviction that abortion is wrong, one should still believe that abortion is wrong even if an authority figure states it is permissible (Nucci, 2001; Nucci \& Turiel, 1978; Turiel, 1983). Finally, people treat their moral beliefs as if they are readily observable, objective facts about the world, much like scientific facts (Shweder, 2002); this perceived objectivity motivates people to defend their moral beliefs and feel justified for doing so (Skitka, Bauman \& Mullen, 2008; Smith, 1994). Consistent with this notion, people often respond to moral transgressions by expressing moral outrage, characterized by making negative attributions about the transgressor, expressing negative affect, and punitiveness (Skitka, Bauman \& Mullen, 2004; Tetlock, Kristel, Elson, Green \& Lerner, 2000).

Given the perceived objectivity and universality of moral conviction and that moral conviction can be authority independent, we hypothesize that MMs could influence not only people's perceptions of fairness, but also their subsequent willingness to flout rules and engage in deviant behavior. In particular, we predict that when outcomes are inconsistent rather than consistent with moral standards, people will be more likely to flout rules and engage in deviant behavior.

\section{Procedural Fairness}

At first glance, our hypothesis that whether outcomes support or oppose moral standards will predict whether people engage in deviant behavior seems inconsistent with research that suggests that procedural elements are the best predictors of people's willingness to obey the law (Tyler, 1990). For example, considerable research on the fair process effect has demonstrated that positive aspects of procedures positively influence people's perceptions of procedural and distributive fairness, their decision acceptance, and their willingness to obey authorities' rules 
(e.g., Folger, Rosenfeld, Grove, \& Corkran, 1979; Greenberg \& Folger, 1983). Indeed, some research suggests that people will accept negative or unfavorable outcomes as fair when they are arrived at through fair procedures (e.g., Van den Bos, Lind, Vermunt, \& Wilke, 1997). We argue, however, that the fair process effect is subject to important boundary conditions. That is, fair process might predict willingness to accept outcomes when those outcomes are merely unfavorable. When outcomes are inconsistent with moral standards, however, then the fairness of the process might become less important. In particular, in domains where people have a clear sense of what constitutes a moral or just outcome, we argue that moral conviction will have a stronger influence than procedural fairness on perceptions of outcome fairness and deviant behavior. This is because moral conviction leads to certainty about what outcomes authorities and procedures should deliver. Outcomes perceived to have moral implications can therefore serve as a check on whether institutional systems or authorities responsible for the decision are just and legitimate (Skitka, Bauman \& Mullen, 2008). “Wrong” answers (i.e., outcomes that oppose people’s moral standards) signal to perceivers that the system is flawed and possibly illegitimate. In these circumstances, failure on the part of an authority or institution to arrive at the correct moral outcome should lead to a loss of faith in authorities and the system itself, even when perceivers would consider the same procedures to be fair when used in a non-moral context in which the "right" decision is less certain (Skitka, 2006).

\section{Moral Spillovers}

In the current research, we investigated moral spillovers - that is, the behavioral consequences of experiencing a MM violation. In particular, we investigated whether people were more likely to engage in deviant behavior when outcomes violated rather than supported their MMs. Consistent with the notion that people are more likely to engage in deviant behavior 
following instances of perceived injustice, theorists have argued that when people are exposed to laws that they perceive to be unjust, people’s support for and compliance with authorities will erode (Robinson \& Darley, 1995). In particular, the flouting hypothesis (Nadler, 2005) predicts that perceived injustice can lead to diminished general compliance with the law and this noncompliance can manifest itself in subtle ways (e.g., littering) unrelated to the perceived unjust law. Supporting the flouting hypothesis, Nadler (2005) found that people’s perceptions that a particular law was unjust led to greater self-reported willingness to engage in lawbreaking (e.g., petty theft) in a subsequent, unrelated context. In the current research, we extend previous theorizing and research by investigating whether outcomes that violate MMs influence not only people’s perceptions of fairness, but also their tendency to engage in deviant behavior.

The goal of the studies described in this paper was to test whether exposing people to “immoral” outcomes would increase their deviant behavior. In Study 1, participants read a newspaper article about a defendant accused of committing a crime that furthered pro-choice beliefs. We manipulated the propriety of the trial procedures and whether the defendant was convicted. We hypothesized that participants would be more likely to (a) judge outcomes to be unfair, (b) reject decisions, and (c) be angered with outcomes, when the verdict in the case opposed rather than supported their MM. More importantly, we further hypothesized that MM violations would increase participants’ likelihood of engaging in deviant behavior (i.e., taking a borrowed pen). In Study 2, we demonstrate that deviant behavior associated with MM violations is specifically a result of witnessing or learning about immoral outcomes, and not simply a result of general anger. 
Study 1

Method

\section{Participants and Design}

One hundred thirty-seven undergraduates (88 women) participated in exchange for $\$ 10$. Participants ranged in age from 18 to $37(M=20.40, S D=1.63)$. We employed a 2 (MM: prochoice, no MM) by 2 (procedural propriety: proper, improper) by 2 (verdict: acquit, convict) between subjects design.

\section{Procedure}

Participants were seated in groups of 20-50 in a large classroom. At each seat was a pen and an envelope containing experimental materials. Participants read a newspaper article that summarized the legal trial of a doctor who allegedly provided an unlawful late-term abortion. Participants were randomly assigned to read about proper or improper trial procedures. In the improper procedures condition, participants read that: (a) the prosecutor violated a gag order and spoke to the press about the defendant in a derogatory manner, (b) the jury was stacked with jurors who opposed the defendant's position on abortion, and (c) the judge blocked the defendant from making a closing statement. Note that all of the improper procedures were biased against the defendant. In the proper procedures condition, the prosecutor abided by the gag order, the jury's views were evenly balanced, and the defendant made a closing statement. Participants were randomly assigned to read that the defendant was found 'Guilty' or 'Not Guilty'. Participants then provided their affective reactions, fairness perceptions, and decision acceptance (see below), and completed two unrelated studies. After participants completed all the studies, they were instructed to return their pen and an envelope containing their materials to two clearly 
labeled, adjacent boxes (one labeled 'PENS', the other labeled 'ENVELOPES') that were located in one corner of the classroom.

\section{Measures}

Moral mandates. One week before the experimental session, participants completed a questionnaire that assessed their attitudes about abortion. Participants reported the extent that they supported or opposed allowing abortion to remain a legal option in the United States on a (3) strongly support to (+3) strongly oppose scale, and answered four items measuring their moral convictions about abortion (see Mullen \& Skitka, 2006, Study 2). For example, participants were asked the extent that they agreed or disagreed with the following item: "My attitude about abortion is closely related to my core moral values and convictions”. Participants' responses to the four items were averaged to create a composite index of moral conviction (Cronbach's $\alpha=$ $.78)$.

Participants were classified as having a "pro-choice MM" if they moderately or strongly supported allowing legal abortion and if they scored at or near the endpoint on the moral conviction scale (indicating moderate to strong agreement with the moral conviction items; Mullen \& Skitka, 2006, Study 2). Likewise, “pro-life MM” participants moderately or strongly opposed abortion and scored at or near the endpoint on the moral conviction scale. All other participants were classified as having "no MM". Note that despite concerted efforts to recruit participants with a pro-life MM, on the campus where we conducted the study there were simply too few student volunteers with pro-life beliefs to fill the cells of our design. Thus, 20 participants who had a pro-life MM were discarded from analyses, leaving 117 participants.

Anger. After reading the trial description and outcome, participants rated how angry they were with the verdict on a 1 (not at all) to 5 (extremely) scale. 
Outcome Fairness. Three items assessed perceived outcome fairness (7-point bipolar scales): (1) “How fair or unfair was the verdict?” (2) “How just or unjust was the verdict?” (3) "How satisfied or dissatisfied are you with the verdict?” Responses were averaged (Cronbach's $\alpha=$ $.92)$.

Decision Acceptance. Participants answered one question designed to tap their decision acceptance (7-point bipolar scale): "How willing or unwilling are you to accept the verdict in the case?”.

Manipulation Checks. To check the verdict manipulation, participants were asked whether the defendant was found guilty. To check the procedural propriety manipulation, participants answered two questions (7-point bipolar scales): (1) "How fair or unfair was the defendant's trial?" (2) "How biased or unbiased were the procedures used in the trial?" Responses were averaged $[r(115)=.68, p<.001]$.

Pen Taking. We unobtrusively numbered identical pens with ink that was only visible under ultraviolet light. Thus, participants did not know that their pen was numbered but the experimenter was able to identify missing pens at the end of each experimental session. Pens left in the box or anywhere in the room were coded as present; pens absent from the room were coded as missing.

$$
\text { Results }
$$

\section{Manipulation Checks}

Two participants incorrectly recalled the verdict and were therefore excluded from analyses. Including versus excluding these participants did not change the pattern of results. In addition, the procedural propriety manipulation was effective: results of a one-way ANOVA revealed that 
participants rated proper procedures to be more fair $(M=0.72, S D=1.07)$ than improper

procedures $(M=-1.29, S D=0.99), F(1,113)=109.73, p<.001, \eta^{2}=.49$.

\section{Outcome Fairness}

Results of a 2 (MM: pro-choice, no MM) by 2 (procedural propriety: proper, improper) by 2 (verdict: acquit, convict) ANOVA with outcome fairness as the dependent measure revealed a significant main effect for verdict, $F(1,107)=20.37, p<.001, \eta_{\mathrm{p}}{ }^{2}=.16$, that was qualified by the predicted MM by verdict interaction, $F(1,107)=4.68, p=.033, \eta_{\mathrm{p}}{ }^{2}=.04$, see Table 1 . Follow-up analysis revealed that participants rated an acquittal to be more fair than a conviction, but this effect was larger for pro-choice MM participants, $F(1,107)=14.68, p<.001, \eta_{\mathrm{p}}{ }^{2}=.31$, than for non-mandated participants, $F(1,107)=6.08, p<.025, \eta_{\mathrm{p}}{ }^{2}=.07$. In particular, prochoice MM participants rated an acquittal to be particularly fair and a conviction to be unfair, irrespective of whether procedures were proper or improper. None of the other interactions or main effects reached statistical significance, all p’s > .16.

\section{Decision Acceptance}

Results of a 2 (MM: pro-choice MM, no MM) by 2 (procedural propriety: proper, improper) by 2 (verdict: acquit, convict) ANOVA with decision acceptance as the dependent measure revealed a significant main effect for verdict, $F(1,107)=20.36, p<.001, \eta_{\mathrm{p}}{ }^{2}=.16$, that was qualified by the predicted MM by verdict interaction, $F(1,107)=12.07, p<.001, \eta_{\mathrm{p}}{ }^{2}=.10$, see Table 1. Follow-up analyses revealed that there was not a significant effect of verdict on decision acceptance when participants did not have a MM, $F(1,107)=1.81$, $n s$. In contrast, prochoice MM participants were more willing to accept the verdict when the defendant was acquitted than when he was convicted, $F(1,107)=21.73, p<.001, \eta_{\mathrm{p}}{ }^{2}=.44$. Thus, pro-choice MM participants accepted the outcome when it was consistent with their MM and rejected the 
outcome when it was inconsistent with their MM. In contrast, participants without a MM were equally willing to accept the outcome irrespective of the verdict in the case. None of the other interactions or main effects reached statistical significance, all p’s > .13.

Anger

Results of a 2 (MM: pro-choice MM, no MM) by 2 (procedural propriety: proper, improper) by 2 (verdict: acquit, convict) ANOVA with participants’ anger as the dependent measure revealed a significant main effect for verdict, $F(1,107)=11.03, p=.001, \eta_{\mathrm{p}}{ }^{2}=.09$, that was qualified by the predicted MM by verdict interaction, $F(1,107)=4.61, p=.034, \eta_{\mathrm{p}}{ }^{2}=.04$, see Table 1. Follow up analyses revealed that participants without a MM were not differentially angry as a function of verdict, $F(1,107)=1.32, p=.268$. In contrast, pro-choice MM participants were more angered when the defendant was convicted than acquitted, $F(1,107)=$ 8.71, $p=.003, \eta_{\mathrm{p}}{ }^{2}=.21$. Thus, as predicted, pro-choice MM participants expressed some anger when the verdict was inconsistent with their MM, but very little anger when the verdict was consistent with their MM. None of the other interactions or main effects reached statistical significance, all p’s > .13.

\section{Pen Taking}

Figure 1 presents the percentage of pro-choice MM and non-mandated participants who took our pen as a function of verdict. Given that pen taking and the independent variables were categorical, logit modeling was used to test the hypothesis that verdict and MM would interact to influence pen taking. In logit modeling, the unit of analysis is the sample cell frequencies (rather than the individual participants). In evaluating logit models, three issues must be considered. First, the overall goodness of fit of the model must be evaluated with the likelihood ratio chisquare test. A nonsignificant chi-square is desired, indicating that there was no significant 
difference between the hypothesized model and the data (i.e., the data fit the model). Note that the hypothesized model is compared to the saturated model (i.e., a model that includes all the possible main and interactive effects of the specified variables). Given that the saturated model will always perfectly reproduce the sample cell frequencies, the aim of evaluating logit models is to determine the most parsimonious, theoretically derived model that is not significantly different from the saturated model. Thus, when there is no difference between the hypothesized model and the saturated model (indicated by a nonsignificant LR chi-square), the hypothesized model is preferred for parsimony. Second, once a parsimonious, well-fitting model is identified, then the significance of each predictor in the model must be evaluated. It is desirable that the hypothesized predictors are statistically significant. Finally, it is important to examine whether any significant two-way interaction explains unique variance beyond that accounted for by the main effects. This is accomplished by comparing models that include versus exclude the significant two-way interaction (i.e., excluding the two-way interaction should significantly decrease model fit; Demaris, 1992).

The moral mandate model. We hypothesized that MM and verdict would interact to influence pen taking and that this effect would hold beyond any main effect of procedural propriety. To test this hypothesis, we evaluated the fit of a model containing the main effects of procedural propriety, verdict and MM and the predicted verdict by MM interaction. The likelihood ratio chisquare test was nonsignificant, indicating that the data fit the model: $\operatorname{LR} \chi^{2}(3, N=115)=1.28, p$ $=.73$. In addition, the nonsignificant LR chi-square test indicates that the more parsimonious hypothesized model is preferred over the saturated model (i.e., the model with all main effects, 2-way and 3-way interactions). Said differently, our hypothesized model is doing as good of a job of predicting pen taking as a model that includes the three way interaction between MM, 
procedural propriety and verdict; for parsimony, our hypothesized model is preferred over the saturated model.

Tests of the individual predictors in the model revealed that the main effects of procedural propriety and MM were not significant ( $p=.093$ and .067, respectively). However, results revealed a significant main effect for verdict (parameter coefficient $=-3.05, Z=-1.96, p=.05$ ) that was qualified by the predicted significant interaction between verdict and MM (parameter coefficient $=4.27, Z=2.36, p=.018)$. Following the recommendation of Demaris $(1991,1992)$ we evaluated whether the two-way interaction explained unique variance not captured by the main effects only model. To do this, we evaluated the fit of a model containing only the main effects of MM, verdict, and procedural propriety. The likelihood ratio chi-square test for the main effects only model was significant $\operatorname{LR} \chi^{2}(4, \mathrm{~N}=115)=14.093, p=.007$, indicating a poor fit to the data. Moreover, the chi-square difference test comparing the main effects only model with the predicted model was significant, LR dif $\chi^{2}(1)=12.81, p<.01$, indicating that the interaction explained unique variance not captured by the main effects only model.

Chi-square analyses were used to follow up the significant MM by verdict interaction. Results revealed that having a MM increased pen taking when the defendant was convicted, $\chi^{2}(1$, $\mathrm{N}=40)=4.29, p=.038$. In particular, when the defendant was convicted, $25 \%$ of pro-choice MM participants took our pen whereas only $4 \%$ of non-mandated participants took our pen, see Figure 1. Thus, receiving an outcome that was inconsistent with their moral standards led prochoice MM participants to engage in more deviant behavior relative to non-mandated participants. Results of a chi-square analysis also revealed that having a MM decreased pen taking when the defendant was acquitted, $\chi^{2}(1, \mathrm{~N}=75)=4.60, p=.032$. In particular, when the pro-choice defendant was acquitted, 15\% of non-mandated participants took our pen whereas, 
$0 \%$ of pro-choice MM participants took our pen, see Figure 1. Thus, receiving an outcome that is consistent with one’s moral standards can also decrease deviant behavior.

We also tested whether verdict was related to pen taking for pro-choice MM and nonmandated participants. As predicted, results revealed that pro-choice MM participants were more likely to take our pen when the defendant was convicted (25\%) than when he was acquitted $(0 \%), \chi^{2}(1, N=40)=7.57, p=.006$. In contrast, for participants without a MM, we observed no significant differences in likelihood of taking our pen as a function of whether the defendant was acquitted $(15 \%)$ or convicted $(4 \%), \chi^{2}(1, \mathrm{~N}=75)=2.36, p=.124$.

The procedural justice model. Theories of procedural justice would predict that procedural propriety and possibly the procedural propriety by verdict interaction would be the best predictors of pen taking. To test the procedural justice model we evaluated the fit of a model that contained the main effects of procedural propriety, verdict, MM, and the interactive effect of procedural propriety and verdict. The likelihood ratio chi-square test was significant, indicating a poor fit to the data: $\operatorname{LR} \chi^{2}(3)=14.09, p=.001$. Moreover, tests of the individual predictors in the model revealed that none of the individual predictors in the model were significant (all $p \mathrm{~s}>$ .459). Thus, the effects for procedural propriety and the procedural propriety by verdict interaction were not significant.

Anger as a mediator. One could question whether our results for pen-taking were simply due to the increased anger that participants experienced when they learned about outcomes that were inconsistent with their MMs. Thus, we tested whether anger was a significant predictor of pen taking. Results of a logistic regression with participants' anger as the predictor variable and pen taking as the dependent variable, revealed that anger was not a significant predictor of pen 
taking, Odds Ratio $=1.32$, Wald test $=0.95, p=.341$. Given that anger was not a significant predictor of pen taking, anger could not mediate the effect of MM and verdict on pen taking.

\section{Study 1 Discussion}

Results of Study 1 revealed that pro-choice MM participants were more likely to (a) be angered by the outcome (b) rate the outcome as less fair (c) reject the outcome and (d) walk away with our pen at the end of the experiment when the outcome of the case opposed rather than supported their MM. Indeed, pen taking was highest when pro-choice MM participants learned about an outcome that was inconsistent with their moral standards and was lowest when pro-choice MM participants learned about an outcome that was consistent with their moral standards. Thus, these results provide novel evidence of moral spillover; that is, outcomes that are inconsistent with people’s MMs increase their likelihood of engaging in deviant behavior. Moreover, results of Study 1 also suggest that outcomes that are consistent with moral standards decrease the likelihood of engaging in deviant behavior. Thus, whether outcomes are consistent or inconsistent with moral standards influences people's tendency to engage in deviant behavior. It is also worth noting that the influence of MM and verdict on deviant behavior could not be accounted for by the increased anger participants felt after learning about outcomes that were inconsistent with their MMs.

Study 2

Study 2 was designed to extend the results of Study 1 using a different type of moral violation and a different operationalizaion of deviant behavior (i.e., cheating). In addition, Study 2 was designed to further rule out the possibility that the increased deviant behavior we observed when outcomes opposed moral standards in Study 1, could be attributed to participants' anger rather than the moral violation per se. In Study 2, we first manipulated whether participants 
recalled a moral violation, a time when they were angry or their typical Tuesday (neutral condition). We then provided participants with an opportunity to randomly assign themselves to one of two experimental conditions by flipping a coin. Prior to flipping the coin, participants were told that if their coin landed on tails they would have an opportunity to earn additional experimental pay; whereas if their coin landed on heads they would not earn any additional money. Thus, it was more desirable for the coin to land on tails than on heads. If participants honestly report the result of their coin toss then one would expect the coin to land on tails about $50 \%$ of the time. In contrast, if participants cheat to assign themselves to the condition that allows them to earn additional money, then one would expect participants to report that the coin landed on tails significantly more than $50 \%$ of the time. We predicted that participants in the moral violation condition would be more likely to cheat relative to participants in the neutral or angry conditions. Thus, we expected to find evidence of cheating for participants in the moral violation condition but not for participants in the neutral or anger conditions.

\section{Method}

\section{Participants}

One hundred eight people (70 women) ranging in age from 18 to $70(M=32.75, S D=$ 11.60), participated in the study in exchange for a guaranteed $\$ 4$, and an opportunity to earn an additional \$2 during the course of the study. Participants were recruited from an email list, maintained by a private California university, of individuals interested in participating in online research studies. Fifteen percent of participants reported having a high school diploma, 30\% of participants were current college students, 25\% of participants had a college degree and the remainder of participants had completed some graduate education. Two participants (one in the control condition and one in the moral violation condition) reported suspicion about the coin toss 
procedure and were therefore excluded from analyses, leaving 106 participants. Including versus excluding these participants did not change our results.

\section{Procedure}

After signing up to participate in the study, participants were emailed a link to the study website. After linking to the site, participants completed two short “unrelated” studies. In the

first study, participants were told that the researchers were conducting a study on people’s memory for events. Participants were randomly assigned to one of three conditions: anger, moral violation, control. Participants in the anger condition were asked to recall and write about a time when they were trying to accomplish a task or get something done and they got angry because they could not do what they wanted to do. Participants in the moral violation condition were asked to recall and write about a time when they observed or heard about someone who did something immoral that made them angry. Participants in the control condition were asked to write about their typical Tuesday.

After completing their essay, participants were invited to participate in a second study, the Trivia Game Study. Participants were informed that the researchers in Study 2 were interested in studying the effects of incentives on task performance. Thus, participants were asked to randomly assign themselves to one of two incentive conditions by the flip of a coin. Participants were told that if their coin landed on heads that they would not earn any additional money for each correct trivia answer they provided, whereas if their coin landed on tails they would earn an additional \$0.20 for each correct trivia response. Participants learned that there would be 10 trivia questions, thus they had an opportunity to earn up to an additional \$2 for participating in the Trivia Game Study. After reading all the instructions, participants were asked to flip a coin 
and report the result of their coin toss. Their report of their coin toss result served as our dependent variable.

After reporting their coin toss result, participants answered the 10 trivia questions, and then reported their current mood. In particular, participants were asked to what extent they felt angry, cheerful, and relaxed on 1 (not at all) to 5 (extremely) scales. Participants then provided their demographic information and answered two questions designed to probe their suspicion about using a coin toss to randomly assign themselves to an incentive condition. Participants were then fully debriefed and paid for their participation. To ensure fairness, all participants received a total of $\$ 6$ for participating in the study.

\section{Results and Discussion}

We investigated whether the proportion of people indicating that their coin landed on tails (the money option) was significantly different from chance for each of the three conditions. Descriptive analyses revealed that $60.5 \%$ of participants in the control condition, $63.2 \%$ of participants in the anger condition, and $76.0 \%$ of participants in the moral violation condition reported that their coin landed on tails, see Figure 3. Results of a one-sample t-test that compared whether these percentages were significantly different from $50 \%$ revealed that only participants in the moral violation condition differed significantly from chance, $t(26)=2.98, p=.006$. In contrast, participants in the anger and control conditions were not significantly more likely than chance to report tails, $t(37)=1.66, p=.106$ and $t(42)=1.39, p=.173$, respectively.

Importantly, results of a one-way ANOVA with condition as the independent variable and participants' anger as the dependent variable, revealed that participants did not significantly differ as a function of condition in their self-reported anger at the end of the experiment, $F(2$, $102)<1$. Thus, participants in the anger and moral violation conditions were equally angered at 
the end of the experiment. In summary, results of Study 2 provide additional evidence that violations of moral standards (and not just anger per se) led to increased deviant behavior. Participants who recalled a moral violation were more likely to cheat relative to participants in the angry and neutral conditions. Moreover, these results extend Study 1 by investigating a different operationalization of deviant behavior (i.e., cheating) and utilizing a different type of moral violation.

\section{General Discussion}

The current research extends previous work on MMs by exploring the broader consequences of threats to MMs. Consistent with prior research, in Study 1 we found that participants judged outcomes to be less fair, were less willing to accept outcomes, and were more angered when outcomes opposed rather than supported their MMs. Moreover, the interactive effects of MM and verdict on perceptions of outcome fairness, decision acceptance and anger were not qualified by whether procedures were proper or improper. More importantly, we uncovered novel evidence of a moral spillover effect, by showing that people are more willing to engage in deviant behavior (i.e., take our pen or cheat) when outcomes oppose rather than support their MMs (Study 1), or when simply asked to think about another person violating a moral standard (Study 2). Taken together, our results suggest that outcomes or events that violate moral standards can increase people’s tendency to engage in deviant behavior.

Why are people more willing to engage in deviant behavior after learning about outcomes that violate their MMs? There are a number of processes that can contribute to the relationship between MM violations and deviant behavior. First, it might be that having a MM about what constitutes a fair outcome allows perceivers to check whether authorities and institutions are just and legitimate by assessing whether those institutions produce the "right” outcome. When 
institutions and authorities fail to uphold people’s MMs, they perceive those institutions and authorities to be less legitimate (Skitka, 2006). This decreased legitimacy might lead people to be more willing to engage in deviant behavior and flout the rules of authorities. In other words, when the system seems unjust because moral standards are not upheld, following rules in general, becomes less important.

Another possible mechanism involves focusing participants' attention on the fact that moral violations happen. In the experiments reported here, some participants focused their attention on specific instances of MM violations, and some participants did not (either because they did not have a relevant $\mathrm{MM}$ or because they were not asked to recall a moral violation). By focusing some participants' attention on the fact that moral violations do occur in the world, these participants may have inferred that moral violations happen with some frequency, and so engaging in a moral violation oneself may have been perceived as consistent with a general descriptive norm (cf. Cialdini, Kallgren, \& Reno, 1990). Similarly, being primed with the notion that moral violations occur with some frequency, may have increased participants' tendency to engage in deviant behavior through a less conscious process (e.g., Bargh, Chen \& Burrows, 1996). Like Bargh’s participants who walked more slowly after being primed with stereotypes of the elderly, our participants may have behaved more unfairly after being primed with moral violations.

Irrespective of whether the increased deviant behavior we observed following exposure to a moral violation was the result of a conscious decision to engage in deviant behavior or resulted from a more automatic process, it seems clear that the extent to which authorities, systems and individuals are perceived as upholding moral standards can have a profound influence on people's tendency to comply with rules. When moral standards are not upheld, people are more 
likely to engage in deviant behavior. This suggests that witnessing injustice (through a violation of a moral standard) can ironically lead people to behave more unfairly themselves (Robinson \& Darley, 1995; Robinson \& Darley, 2007; Nadler, 2005).

The notion that people can feel justified in behaving in an immoral fashion because of experiencing past unfairness is consistent with research on "equity with the world” (Austin \& Walster, 1975). Austin and Walster (1975) theorized that if people feel unfairly treated in one instance, they can be motivated to maintain equity with the world by recouping their losses in a later instance, even when the two instances are unrelated and involve interactions with different people or institutions. For example, participants who were underpaid in a first task were especially likely to try to recoup their losses in a subsequent task (i.e., by taking more than their “fair” share) when they felt more anonymous - that is, when they would not have to meet with their task partner (Austin \& Walster, 1975). Thus, receiving an unfair allocation led participants to behave more unfairly in a subsequent task when anonymity was high but not when anonymity was low. This research suggests that the perceived anonymity we provided our participants may have increased their willingness to engage in deviant behavior. In the first experiment, participants did not know that the pens were unobtrusively marked, and in the second experiment, participants knew that the experimenters were unable to verify their coin flip results. Thus, participants had every reason to believe that their deviant behavior would go undetected. Future research should therefore examine whether anonymity moderates the effects of moral violations on deviant behavior.

In addition, future research should also investigate whether there might be prosocial consequences of outcomes that affirm people’s MMs. Results of Study 1 suggest that affirmations of MMs (e.g., when those who are on trial for "moral” actions are set free) might 
lead people to be more willing to adhere to authorities’ rules. In Study 1, pro-choice MM participants did not take any pens when the outcome supported their MM. Future research should therefore continue to explore how outcomes that support or oppose moral standards can lead to more versus less adherence with rules, respectively.

In addition, future research should test the MM hypothesis with a more representative sample of the population. Because we could not recruit enough pro-life MM participants in Study 1, one might worry that our results are limited to explaining the responses of pro-choice MM participants. However, other research has found no statistical differences in the fairness judgments of pro-life and pro-choice participants following a violation of a MM (Mullen \& Skitka, 2006), and has found evidence for the MM effect using representative samples of Americans across a variety of topical domains (Skitka, 2002, 2006). In addition, in Study 2 we found evidence of increased deviant behavior following a moral violation using adult participants that represented a broader sample of the population (i.e., participants had a broader range of educational backgrounds and age relative to the liberal college students used in Study 1). Thus, it seems reasonable to conclude that our moral spillover results generalize beyond pro-choice MM participants and are not simply a tendency displayed by liberal college students, but future research should nevertheless demonstrate this empirically.

In conclusion, we observed very different patterns of behavior depending on whether moral convictions were implicated. Across both studies, exposure to morally threatening outcomes increased the likelihood of engaging in deviant behavior. In addition, in Study 1, exposure to morally affirming outcomes decreased the likelihood of engaging in deviant behavior. At the same time, we observed no significant difference in likelihood of deviant behavior when the outcome was unrelated to participants’ MMs, even when participants perceived the outcome to 
be unfair (Study 1) or anger-provoking (Study 2). Moreover, in Study 1, we observed no significant effects of procedural fairness on people's perceptions of outcome fairness, decision acceptance, anger, or deviant behavior. Although previous research suggests that people are more willing to accept unfavorable outcomes and comply with authorities' rules when procedures are fair (e.g., Folger, et al., 1979; Tyler, 1990), our results suggest that the fair process effect may be weaker when people have a MM relevant to the issue at hand. In conclusion, the extent to which authorities and institutions produce morally tenable outcomes may influence people's tendency to follow the rules. Our results suggest that there are limits to authorities' ability to gain citizen cooperation and compliance with rules when outcomes are inconsistent with people's moral standards. 


\section{References}

Austin, W. \& Walster, E. (1975). Equity with the world: The trans-relational effects of equity and inequity. Sociometry, 38, 474-495.

Bargh, J. A., Chen, M., \& Burrows, L. (1996). Automaticity of social behavior: Direct effects of trait construct and stereotype activation on action. Journal of Personality and Social Psychology, 71, 230-244.

Bauman, C. W. (2007). Procedural and moral influences on fairness judgments, decision acceptance, and organizational behavior (Doctoral Dissertation, University of Illinois at Chicago, 2006). Dissertation Abstracts International: Section B: The Sciences and Engineering, 67, 5463.

Cannon, L. (1999). Official negligence: How Rodney King and the riots changed Los Angeles and the LAPD. Boulder, CO: Westview Press.

Cialdini, R. B., Reno, R. R., \& Kallgren, C. A. (1990). A focus theory of normative conduct: Recycling the concept of norms to reduce littering in public places. Journal of Personality and Social Psychology, 58, 1015-1026.

Demaris, A. (1991). A framework for the interpretation of first-order interaction in logit modeling. Psychological Bulletin, 110, 557-570.

Demaris, A. (1992). Logit modeling: Practical applications. Newbury Park, CA: Sage.

Folger, R., Rosenfeld, D., Grove, J. \& Corkran, L. (1979). Effects of “voice” and peer opinions on responses to inequity. Journal of Personality and Social Psychology, 37, 2253-2261.

Friedman, L. (1975). The legal system: A social science perspective. NY: Russell Sage Foundation. 
Greenberg, J. \& Folger, R. (1983). Procedural justice, participation and the fair process effect in groups and organizations. In P. B. Paulus (Ed.), Basic group processes (pp. 235-256). NY: Springer Verlag.

Haidt, J., Rosenberg, E., \& Hom, H. (2003). Differentiating diversities: Moral diversity is not like other kinds. Journal of Applied Social Psychology, 33, 1-36.

Hare, R. M. (1981). Moral thinking. Oxford, England: Oxford University Press.

Kant, I. (1947). Fundamentals of the metaphysics of morals. New York: Longmans. (Original work published 1786).

Kohlberg, L. (1984). Essays on moral development: Vol. 2. The psychology of moral development. New York: Harper.

Mullen, E., \& Skitka, L. J. (2006). Exploring the psychological underpinnings of the moral mandate effect: Motivated reasoning, group differentiation, or anger? Journal of Personality and Social Psychology, 90, 629-643.

Nadler, J. (2005). Flouting the law. Texas Law Review, 83, 1399-1441.

Nucci, L. P. (2001). Education in the moral domain. Cambridge: Cambridge University Press.

Nucci, L. P., \& Turiel, E. (1978). Social interaction and the development of social concepts in pre-school children. Child Development, 49, 400-407.

Petty, R. E. \& Krosnick, J. A. (1995). Attitude strength: An overview. Attitude strength: Antecedents and consequences (pp. 1-24). Mahwah, NJ: Lawrence Erlbaum.

Piaget, J. (1932/1997). The moral judgment of the child. Glencoe, IL: Free Press.

Robinson, P. H., \& Darley, J. M. (1995). The utility of desert. Northwestern University Law Review, 91, 453-499. 
Robinson, P. H., \& Darley, J. M. (2007). Intuitions of justice: Implications for criminal law and justice policy. Southern California Law Review, 81 1-67.

Shweder, R. (2002). The nature of morality: the category of bad acts. Medical Ethics, 9, 6-7.

Skitka, L. J. (2002). Do the means justify the ends, or do the ends justify the means? A test of the value protection model of justice. Personality and Social Psychology Bulletin, 28, 588-597.

Skitka, L. J. (2006). Legislating morality: How deep is the U.S. Supreme Court's reservoir of good will? Paper presented at the meeting of the International Society for Justice Research, Berlin, Germany.

Skitka, L. J., Bauman, C. W., \& Mullen, E. (2004). Political tolerance and coming to psychological closure following the September 11, 2001 terrorist attacks: An integrative approach. Personality and Social Psychology Bulletin, 30, 743-756.

Skitka, L. J., Bauman, C. W., \& Mullen, E. (2008). Morality and justice: An expanded theoretical perspective and empirical review. In K. A. Hegtvedt and J. Clay-Warner (Eds.). Advances in Group Processes: Justice.

Skitka, L. J., \& Mullen, E. (2002). Understanding judgments of fairness in a real-world political context: A test of the value protection model of justice reasoning. Personality and Social Psychology Bulletin, 28, 1419-1429.

Smith, M. (1994). The moral problem. Oxford, England: Blackwell.

Tetlock, P. E., Kristel, O. V., Elson, S. B., Green, M. C. \& Lerner, J. S. (2000). The psychology of the unthinkable: Taboo trade-offs, forbidden base rates, and heretical counterfactuals. Journal of Personality and Social Psychology, 78, 853-870.

Tyler, T. R. (1990). Why people obey the law. New Haven, CT: Yale University Press. 
Turiel, E. (1983). The development of social knowledge: Morality and convention. Cambridge, England: Cambridge University Press.

Van den Bos, K., Lind, E. A., Vermunt, R., \& Wilke, H. A. M. (1997). How do I judge my outcome when I do not know the outcome of others? The psychology of the fair process effect. Journal of Personality and Social Psychology, 72, 1034- 1046. 


\section{Acknowledgements}

Address correspondence to Elizabeth Mullen, Stanford University, 518 Memorial Way, Stanford, CA 94305-5015 (e-mail: emullen@stanford.edu). This research was supported by the Dispute Resolution Research Center at Northwestern University, the American Bar Foundation, and was facilitated by National Science Foundation Grant SES-0530380. We thank Gillian Fensterman and Jennifer Rosner for their help with data collection and John Patrick Johnson for his help with the logit analyses in Study 1. 
Table 1.

Perceived Outcome Fairness, Decision Acceptance and Anger with the Verdict as a Function of Moral Mandate and Verdict (Study 1).

\begin{tabular}{|c|c|c|c|c|}
\hline \multirow[b]{3}{*}{ Verdict } & \multicolumn{4}{|c|}{$\underline{\text { Moral mandate }}$} \\
\hline & \multicolumn{2}{|c|}{ Pro-Choice moral mandate } & \multicolumn{2}{|c|}{$\underline{\text { No moral mandate }}$} \\
\hline & $M$ & $S D$ & $M$ & $S D$ \\
\hline \multicolumn{5}{|l|}{ Outcome Fairness } \\
\hline Acquit & 1.54 & 1.30 & 0.69 & 1.46 \\
\hline Convict & -0.33 & 1.38 & -0.14 & 1.48 \\
\hline \multicolumn{5}{|c|}{ Decision Acceptance } \\
\hline Acquit & 2.07 & 1.27 & 0.74 & 1.78 \\
\hline Convict & -0.58 & 1.73 & 0.21 & 1.70 \\
\hline \multicolumn{5}{|c|}{ Anger with the Verdict } \\
\hline Acquit & 1.36 & 0.78 & 1.81 & 0.99 \\
\hline Convict & 2.33 & 1.16 & 2.07 & .98 \\
\hline
\end{tabular}

Note: Fairness ratings ranged from (-3) very unfair to (+3) very fair. Decision acceptance ranged from (-3) very unwilling to (+3) very willing. Anger with the verdict ranged from (1) Not at all to (5) Extremely. 
Figure 1. Percentage of participants engaging in deviant behavior as a function of moral mandate and verdict, Study 1.

\section{Acquit $\square$ Convict}

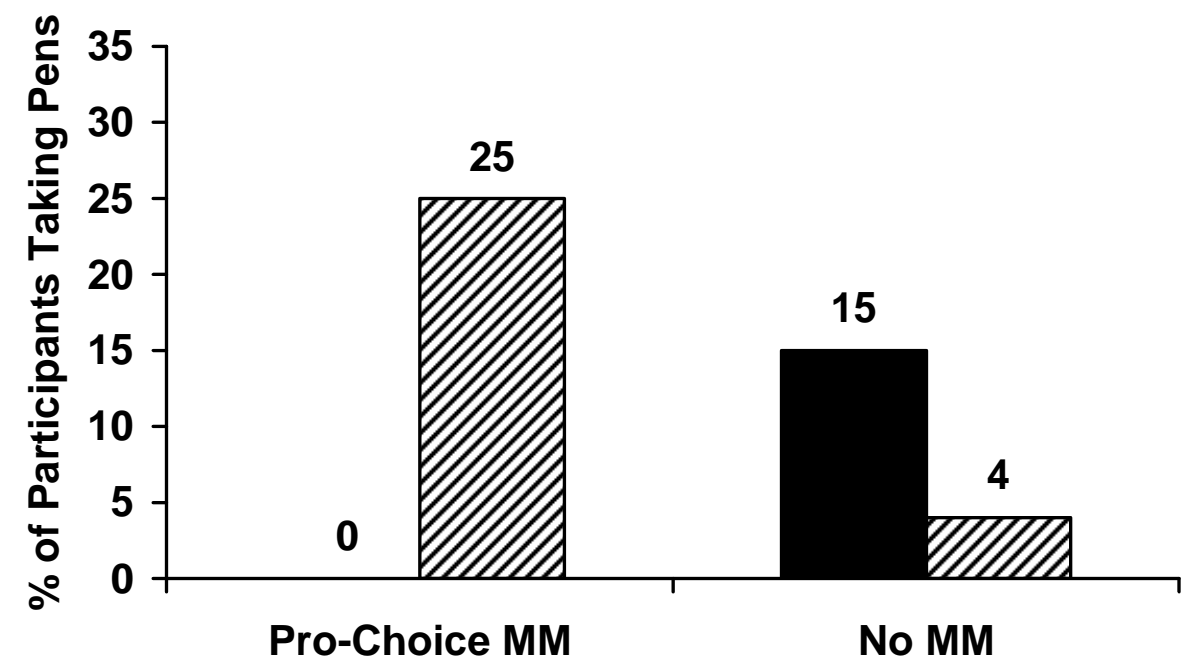


Figure 2. Percentage of participants reporting a favorable coin flip as a function of condition, Study 2.

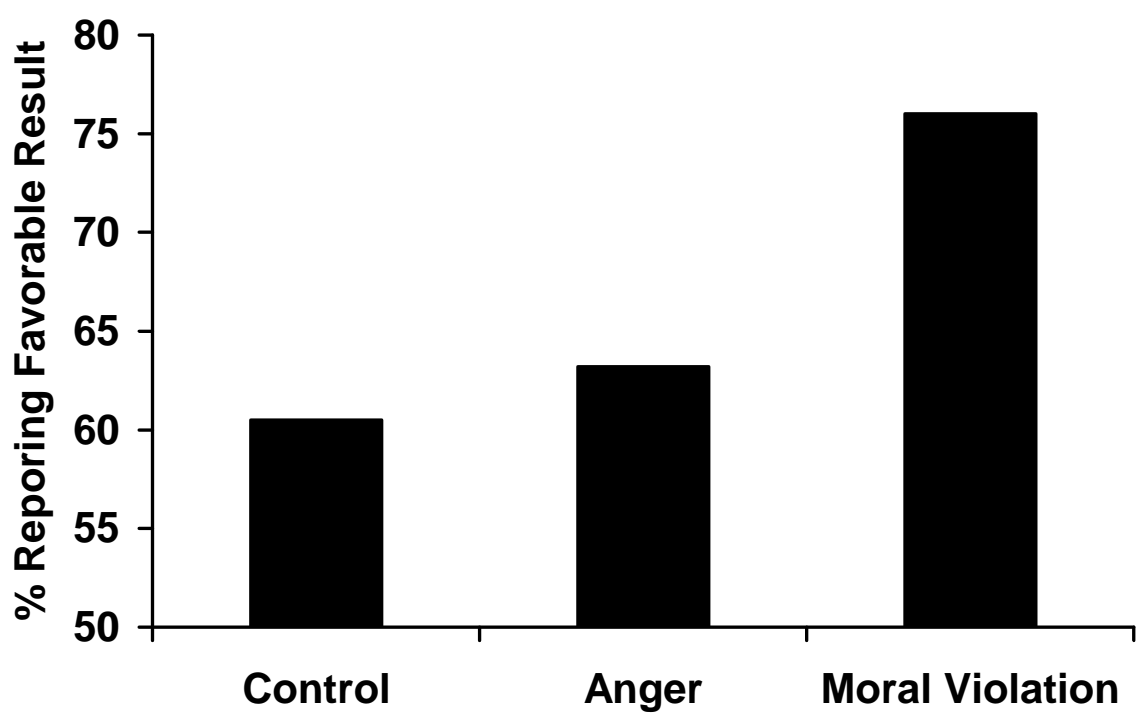

